\title{
Análise genética de escores de avaliação visual de bovinos com modelos bayesianos de limiar e linear
}

\author{
Carina Ubirajara de Faria(1), Cláudio Ulhôa Magnabosco(2), Lúcia Galvão de Albuquerque ${ }^{(3)}$, \\ Arcadio de los Reyes ${ }^{(4)}$, Luiz Antônio Framartino Bezerra ${ }^{(5)}$ e Raysildo Barbosa Lobo ${ }^{(6)}$
}

\begin{abstract}
(1)Universidade Federal de Goiás (UFG), Campus de Jataí, Caixa Postal 03, CEP 75800-000 Jataí, GO. E-mail: carinauf@yahoo.com.br (2)Embrapa Cerrados, Caixa Postal 08223, CEP 73310-970 Planaltina, DF. E-mail: mclaudio@cpac.embrapa.br (3)Universidade Estadual Paulista, Faculdade de Ciências Agrárias e Veterinárias, Departamento de Melhoramento Animal, Via de acesso Prof. Paulo Donato Castellane, s/no, CEP 14884-900 Jaboticabal, SP. E-mail: Igalb@fcav.unesp.br (4)UFG, Escola de Veterinária, Departamento de Produção Animal, Campus Samambaia, Caixa Postal 131, CEP 74001-970 Goiânia, GO. E-mail: adlreyes@vet.ufg.br (5)Universidade de São Paulo, Faculdade de Medicina de Ribeirão Preto, Departamento de Genética, Avenida Bandeirantes, no 3.900, Monte Alegre, CEP 14040-030 Ribeirão Preto, SP. E-mail: Ifbezer@fmrp.usp.br ${ }^{(6)}$ Associação Nacional de Criadores e Pesquisadores, Rua João Godoy, no 463, CEP $14020-230$ Ribeirão Preto, SP. E-mail: raysildo@ancp.org.br
\end{abstract}

Resumo - O objetivo deste trabalho foi comparar as estimativas de parâmetros genéticos obtidas em análises bayesianas uni-característica e bi-característica, em modelo animal linear e de limiar, considerando-se as características categóricas morfológicas de bovinos da raça Nelore. Os dados de musculosidade, estrutura física e conformação foram obtidos entre 2000 e 2005, em 3.864 animais de 13 fazendas participantes do Programa Nelore Brasil. Foram realizadas análises bayesianas uni e bi-características, em modelos de limiar e linear. De modo geral, os modelos de limiar e linear foram eficientes na estimação dos parâmetros genéticos para escores visuais em análises bayesianas uni-características. Nas análises bi-características, observou-se que: com utilização de dados contínuos e categóricos, o modelo de limiar proporcionou estimativas de correlação genética de maior magnitude do que aquelas do modelo linear; e com o uso de dados categóricos, as estimativas de herdabilidade foram semelhantes. A vantagem do modelo linear foi o menor tempo gasto no processamento das análises. $\mathrm{Na}$ avaliação genética de animais para escores visuais, o uso do modelo de limiar ou linear não influenciou a classificação dos animais, quanto aos valores genéticos preditos, o que indica que ambos os modelos podem ser utilizados em programas de melhoramento genético.

Termos para indexação: amostragem de Gibbs, características morfológicas, gado de corte, valores genéticos.

\section{Genetic analysis for visual scores of bovines with the linear and threshold bayesian models}

\begin{abstract}
The objective of this work was to compare the estimates of genetic parameters obtained in singletrait and two-trait bayesian analyses, under linear and threshold animal models, considering categorical morphological traits of bovines of the Nelore breed. Data of musculature, physical structure and conformation were obtained between years 2000 and 2005, from 3,864 bovines of the Nelore breed from 13 participant farms of the Nelore Brazil Program. Single-trait and two-trait bayesian analyses were performed under linear and threshold animal models. In general, the linear and threshold models were efficient in estimating genetic parameters for visual scores under single-trait bayesian analyses. In the two-trait analyses, it was observed that: using continuous and categorical data, the threshold model provided greater estimates of genetic correlation than those of the linear model; with categorical data, the heritability estimates were similar. One major advantage of the linear models was its smaller requirements in the analyses processing time. In the genetic evaluation of animals for visual scores, the use of the linear or threshold model did not influence the classification of the animals, based on their predicted breeding values, which suggests that both models can be used in genetic improvement programs.
\end{abstract}

Index terms: Gibbs sampling, morphological traits, beef cattle, breeding values.

\section{Introdução}

No Brasil, as características morfológicas de conformação de bovinos de corte têm despertado grande interesse em criadores e pesquisadores, e estudos têm sido realizados para se verificar quais são os fatores genéticos e ambientais que afetam essas características.
As pesquisas realizadas indicam que a seleção baseada nos escores de conformação, precocidade e musculatura pode ser efetiva, visto que as estimativas de herdabilidade obtidas são de moderada a média magnitude (Dal Farra et al., 2002; Koury Filho, 2005; Kippert et al., 2006). No entanto, para se obter sucesso com a utilização dos 
escores visuais como critérios de seleção, é necessário conhecer os fatores ambientais e genéticos.

Os parâmetros genéticos têm sido estimados por diferentes métodos, que evoluem à medida que novas teorias e técnicas computacionais são desenvolvidas. O método de estimação é um fator importante na obtenção dos parâmetros genéticos, com o estabelecimento de um modelo que descreva corretamente os dados.

Gianola \& Foulley (1983) desenvolveram o modelo de limiar para avaliação genética de dados categóricos. Posteriormente, Van Tassel et al. (1998) tornaram possíveis as análises multi-características que relacionam dados contínuos e categóricos. Os modelos de limiar admitem que existe uma variável subjacente de distribuição contínua, em relação à variável discreta (Sorensen et al., 1995), e as estimativas referentes a uma determinada categoria são observadas se os valores da escala subjacente estiverem localizados entre os limiares que definem essa categoria. Assim, a distribuição de probabilidade das estimativas, para dados categóricos, depende da posição da média da distribuição subjacente contínua, em relação aos limiares fixos.

O programa MTGSAM Threshold ("multiple trait Gibbs sampler for animal models"), desenvolvido por Van Tassel et al. (1998), que utiliza a inferência bayesiana sob o enfoque da amostragem de Gibbs, tem sido utilizado na estimação de parâmetros genéticos para dados categóricos em modelo de touro e em modelo touro avô materno. Em modelo animal, alguns autores (Moreno et al., 1997; Luo et al., 2001; Phocas \& Laloë, 2003) encontraram dificuldades para atingir a convergência da cadeia de Gibbs, uma vez que o algoritmo utilizado caracteriza-se como um processo iterativo.

Os modelos lineares têm sido preferidos em relação aos de limiar, na avaliação genética de características categóricas, pela facilidade de aplicação tanto em modelo animal quanto no modelo de touro, e pelo menor tempo gasto de processamento. Esses modelos foram utilizados em diversos estudos com dados categóricos, entre os quais se destacam os de Weigel et al. (1999), Carnier et al. (2000), Bennett et al. (2001), Cardoso et al. (2001) e Kippert et al. (2006). No entanto, os procedimentos lineares são descritos como não apropriados para se quantificar a natureza discreta dos dados categóricos (Marcondes et al., 2005). Esse fato implica no problema de se gerarem valores baixos das estimativas dos parâmetros genéticos e em conclusões equivocadas referentes ao ganho genético dessas características, quando submetidas à seleção.
O objetivo deste trabalho foi comparar as estimativas de parâmetros genéticos, obtidas em análises bayesianas uni-característica e bi-característica, em modelo animal linear e de limiar, quanto às características categóricas morfológicas de bovinos da raça Nelore.

\section{Material e Métodos}

Foram estudadas as características categóricas morfológicas musculosidade, estrutura física e conformação; e a característica contínua peso, aos 210 dias de idade (P210), em bovinos da raça Nelore. As informações das características morfológicas foram obtidas entre 2000 e 2005, relativas a 3.864 animais de 13 fazendas participantes do Programa Nelore Brasil, localizadas em Goiás, Mato Grosso, Paraná e Distrito Federal. As informações de P210 foram obtidas de 19.093 animais, pertencentes aos rebanhos avaliados, nascidos entre 1990 e 2004.

Os animais foram avaliados visualmente, pelo método MERCOS, adaptado por Lôbo (1996), em que: na musculosidade, avaliou-se a distribuição muscular no corpo do animal, bem como o seu desenvolvimento, volume e comprimento dos músculos, quanto à precocidade de acabamento e maior rendimento de carcaça; na estrutura física, avaliou-se a sustentação do animal, ou seja, cascos quanto à integridade e tamanho, ligamentos ou articulações, quanto à firmeza, e a largura dos ossos; na conformação, avaliou-se o esqueleto do animal, comprimento do corpo, amplitude torácica, arqueamento e comprimento das costelas, largura de garupa e comprimento, relação tamanho dos membros e comprimento das costelas, em que as linhas dorsal e ventral devem ser paralelas ou abrir levemente no sentido da garupa. De acordo com Lôbo (1996), em cada variável morfológica, os animais avaliados podem obter escores de 1 a 5 pontos, e a maior pontuação representa o grau mais favorável. Na Tabela 1, está apresentada a distribuição dos escores em percentagem para cada característica morfológica.

Tabela 1. Distribuição (\%) por escore das características musculosidade (M), estrutura física (E) e conformação (C), em bovinos da raça Nelore.

\begin{tabular}{ccrccc}
\hline Característica & \multicolumn{5}{c}{ Escores } \\
\cline { 2 - 6 } & 1 & \multicolumn{1}{c}{2} & 3 & 4 & 5 \\
\hline M & 0,6 & 12,4 & 43,2 & 33,4 & 10,4 \\
E & 0,4 & 6,5 & 37,6 & 44,0 & 11,5 \\
C & 0,4 & 11,3 & 39,0 & 35,8 & 13,5 \\
\hline
\end{tabular}


A estruturação dos arquivos de dados foi realizada com a utilização do SAS (SAS Institute, 2004). Os dados analisados continham animais cuja idade variou entre 570 e 750 dias, intervalo determinado de acordo com a idade-padrão de animais jovens, 660 dias (22 meses de idade). Na verificação dos fatores de ambiente, que influenciam as características morfológicas, foram realizadas análises prévias pelos procedimentos GLM e REG do SAS (SAS Institute, 2004).

A definição dos grupos de contemporâneos quanto às características categóricas morfológicas foi estabelecida por fazenda, ano e estação de nascimento e lote de avaliação. Na característica contínua P210, os grupos contemporâneos constituíram-se de fazenda, ano e estação de nascimento, sexo e lote de manejo aos 210 dias. O efeito de estação de nascimento foi dividido em quatro classes para os animais nascidos nos períodos de: janeiro a março, abril a junho, julho a setembro e outubro a dezembro. Foram eliminados os grupos contemporâneos que continham menos de quatro animais para todas as características avaliadas. No peso aos 210 dias de idade, além desta restrição, foram retirados os animais com medidas de 3,5 desvios-padrão, acima ou abaixo da média do seu grupo de contemporâneos, para eliminar possíveis dados discrepantes.

$\mathrm{Na}$ estimação dos parâmetros genéticos, foram consideradas as análises bayesianas uni e bicaracterísticas, em modelos de limiar e linear. Os parâmetros genéticos das características categóricas morfológicas e o P210 foram estimados por meio do aplicativo MTGSAM Threshold.

Quanto às características morfológicas, foi considerado o sexo como efeito fixo, os grupos de contemporâneos como efeitos aleatórios e a idade na data da coleta como covariável (efeito linear). Em relação ao peso aos 210 dias de idade, foram incluídos os efeitos fixos de grupo de contemporâneos e a classe de idade da vaca ao parto. O modelo completo pode ser representado em notação matricial como: $\mathrm{y}=\mathrm{X} \beta+\mathrm{Z}_{1} \mathrm{a}+\mathrm{Z}_{2} \mathrm{c}+\mathrm{e}$, em que: y é o vetor das observações (característica categórica ou contínua); $\beta$ é o vetor dos efeitos fixos (sexo para as características morfológicas, grupo de contemporâneos e classe de idade da vaca ao parto para P210); a é o vetor dos efeitos aleatórios que representam os valores genéticos aditivos diretos de cada animal; c é o vetor de efeitos aleatórios não correlacionados (efeito de grupos contemporâneos para as características morfológicas ou efeito de ambiente permanente materno para P210); e é o vetor de efeitos aleatórios residuais; e $X, Z_{1}$ e $Z_{2}$ são as matrizes de incidência, que relacionam as observações aos efeitos fixos e aos efeitos aleatórios genéticos aditivos diretos e não correlacionados, respectivamente. $\mathrm{O}$ arquivo de genealogia incluiu 52.361 animais da raça Nelore.

Os modelos de limiar, geralmente, apresentam problemas na estimação de componentes de variância e predição dos valores genéticos, quando se tem um grande número de níveis de efeitos fixos (Moreno et al., 1997; Varona et al., 1999; Luo et al., 2002). Nesta condição, os autores recomendam assumir esses efeitos como aleatórios. No entanto, para que a convergência seja atingida, também é necessário que haja maior número de dados dentro de cada nível (Varona et al., 1999). Assim, os efeitos de grupos de contemporâneos foram assumidos como aleatórios, e o sexo como efeito fixo, para as análises genéticas de características categóricas.

No modelo de limiar, assumiu-se que a escala subjacente apresenta distribuição normal contínua, representada como: $\mathrm{U}$ I $\theta \sim \mathrm{N}\left(\mathrm{W} \theta, \mathrm{I}^{2}{ }_{\mathrm{e}}\right)$, em que: $\mathrm{U}$ é o vetor da escala base de ordem r; $\theta^{\prime}=\left(\beta^{\prime}, a^{\prime}, c^{\prime}\right)$ é o vetor dos parâmetros de locação de ordem $s, \operatorname{com} \beta$ definido sob o ponto de vista frequientista como efeitos fixos, e ordem s, com a e c como efeitos aleatórios genético aditivo direto e de grupo de contemporâneos; W é a matriz de incidência conhecida, de ordem r por s; I é a matriz de identidade de ordem $r$ por $r$; e $\sigma^{2}$ é a variância residual. Considerando-se que a variável na distribuição subjacente não é observável, a parametrização $\sigma_{\mathrm{e}}^{2}=1$ é geralmente adotada para se obter identificabilidade na função de verossimilhança (Gianola \& Sorensen, 2002). Tal pressuposição é padrão em análises para dados categóricos em modelo de limiar (Van Tassel et al., 1998).

As características categóricas são determinadas por variáveis contínuas não observáveis, em escala subjacente, em que são fixados valores iniciais de

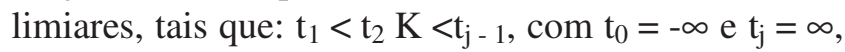
em que j é o número de categorias. Os dados observáveis são dependentes da variável subjacente, que é limitada entre dois limiares não observáveis (Gianola \& Foulley, 1983). Assim, as categorias ou escores de $y_{i}$ (características morfológicas), para cada animal i, são definidos por $U_{i}$, na escala subjacente: 


$$
\mathrm{y}_{\mathrm{i}}=\left\{\begin{array}{l}
1 \mathrm{t}_{0}<\mathrm{U}_{\mathrm{i}} \leq \mathrm{t}_{1} \\
2 \mathrm{t}_{1}<\mathrm{U}_{\mathrm{i}} \leq \mathrm{t}_{2} \\
3 \mathrm{t}_{2}<\mathrm{U}_{\mathrm{i}} \leq \mathrm{t}_{3} \\
4 \mathrm{t}_{3}<\mathrm{U}_{\mathrm{i}} \leq \mathrm{t}_{4} \\
5 \mathrm{t}_{4}<\mathrm{U}_{\mathrm{i}} \leq \mathrm{t}_{5} \quad \mathrm{i}=1, \ldots, \mathrm{n}
\end{array}\right.
$$

em que: n é o número de observações. Após as especificações dos limiares $t_{0}$ a $t_{5}$, é necessário que um deles ( de $_{1} \mathrm{a} \mathrm{t}_{4}$ ) seja ajustado a uma constante arbitrária. Neste trabalho, adotou-se a restrição $t_{1}=0$, tal que o vetor de limiares estimáveis foi definido como:

$$
\mathrm{t}=\left[\begin{array}{l}
\mathrm{t}_{2} \\
\mathrm{t}_{3} \\
\mathrm{t}_{4}
\end{array}\right]
$$

Nas análises bi-características envolvendo categóricas e contínuas, de acordo com o enfoque bayesiano, admitiu-se que as distribuições iniciais dos efeitos aleatórios genéticos, não correlacionados e residuais, seguem distribuição normal multivariada, conforme representado a seguir:

$$
\begin{aligned}
& \mathrm{p}\left(\left[\begin{array}{l}
\mathrm{a}_{1} \\
\mathrm{a}_{2}
\end{array}\right] \mid \mathrm{G}\right) \sim \mathrm{N}\left(\left[\begin{array}{l}
0 \\
0
\end{array}\right], \mathrm{G}=\mathrm{G}_{0} \otimes \mathrm{A}\right), \mathrm{p}\left(\begin{array}{l}
\mathrm{c}_{1} \mid \mathrm{C} \\
\mathrm{c}_{2} \mid
\end{array}\right) \sim \mathrm{N}\left(\left[\begin{array}{l}
0 \\
0
\end{array}\right], \mathrm{C}=\mathrm{C}_{0} \otimes \mathrm{I}\right), \\
& \mathrm{p}\left(\begin{array}{l}
\mathrm{e}_{1} \\
\mathrm{e}_{2}
\end{array} \mid \mathrm{R}\right) \sim \mathrm{N}\left(\left[\begin{array}{l}
0 \\
0
\end{array}\right], \mathrm{R}=\mathrm{R}_{0} \otimes \mathrm{I}\right)
\end{aligned}
$$

em que: $\mathrm{G}_{0}$ é a matriz de variâncias e covariâncias genéticas; $\mathrm{C}_{0}$ é a matriz das variâncias de efeitos não correlacionados; $\mathrm{R}_{0}$ é a matriz de variâncias residuais; $\otimes$ é o operador do produto direto; A é a matriz de parentesco; e I é a matriz identidade.

Assumiram-se distribuições iniciais das (co)variâncias como uma Wishart invertida para os efeitos aleatórios genéticos, não correlacionados e residuais das características estudadas, inclusive a covariância entre elas. Foram definidas distribuições iniciais uniformes tanto para os efeitos fixos quanto para os limiares. O parâmetro $v$ é o grau de liberdade correspondente à distribuição Wishart invertida que indica o grau de confiabilidade da distribuição inicial. Neste trabalho, o parâmetro $v$ utilizado foi de valor zero, ou seja, não refletiu grau de conhecimento sobre os parâmetros (Van Tassel et al., 1998).

$\mathrm{Na}$ implementação da amostragem de Gibbs, nas análises em modelo animal de limiar (uni-característica), modelo animal linear-limiar (bi-característica contínua-categórica) e modelo animal limiar-limiar (bi-característica categórica-categórica), foi utilizado um tamanho de cadeia inicial de 500.000 ciclos, em que os primeiros 100.000 foram descartados, e as amostras foram retiradas a cada mil ciclos, no total de 400 amostras iniciais. Em seguida, foram adicionados 100.000 ciclos a cada rodada, até que as estimativas obtidas na última análise fossem iguais às obtidas na análise anterior. Esse critério foi utilizado com o intuito de se verificar se realmente a convergência foi alcançada. As análises das amostras da correlação serial e da convergência da cadeia de Gibbs foram realizadas com o auxílio do programa Gibanal (Van Kaam, 1998).

Nas análises bi-características em modelo animal de limiar-limiar, isto é, na análise simultânea de duas variáveis categóricas, diferentemente da restrição para o processo anterior, adotaram-se as seguintes restrições, $\mathrm{t}_{1}=0$ e $\mathrm{t}_{2}=1$ (Van Tassel et al., 1998). Nas análises em modelo animal linear (uni-característica) e modelo animal linear-linear, foi utilizado o mesmo programa, tendo-se alterado somente a opção para considerar o modelo como linear (opção zero no módulo mtgsprep.exe).

As soluções dos valores genéticos, obtidas para cada animal, foram transformadas em escala de probabilidade pela função PROBNORM do SAS (SAS Institute, 2004), que resultou em diferenças esperadas na progênie (DEPs), desviadas do valor 0,50 ou 50\%. Essa transformação em escala de probabilidade é realizada, para que a unidade da DEP para os escores visuais fosse dada em percentagem. Neste caso, as DEPs predizem a probabilidade de um reprodutor de deixar descendentes que atinjam o escore máximo para a característica avaliada.

$\mathrm{Na}$ verificação das correlações de posto ou correlações de Spearman, entre os diferentes modelos de análise genética das características morfológicas avaliadas por escores visuais, foi aplicado o procedimento PROC CORR do SAS (SAS Institute, 2004).

\section{Resultados e Discussão}

Foram utilizados tamanhos de cadeia de Gibbs de 500.000 até 1.100 .000 ciclos, na obtenção das estimativas de parâmetros genéticos das características morfológicas musculosidade, estrutura física e conformação, considerando-se análises bayesianas em modelo animal de limiar (uni-característica), modelo animal linear-limiar (bi-característica com dados contínuos e categóricos) e modelo animal limiar-limiar (bi-característica com dados categóricos). Nas análises 
bayesianas em modelo animal linear, o tamanho da cadeia de Gibbs foi de 500.000 ciclos, e não foram necessários ciclos adicionais para atingir a convergência da cadeia amostral.

Observou-se que, para musculosidade, as estimativas de média, moda e mediana da herdabilidade foram maiores, quando se utilizou o modelo de limiar (Tabela 2). Para estrutura física e conformação, as estimativas obtidas pelos dois modelos foram praticamente idênticas. De modo geral, observou-se que os dois modelos apresentaram resultados similares quanto à estimação dos parâmetros genéticos das características morfológicas em análises bayesianas uni-características, pois as regiões de credibilidade se sobrepõem, o que caracteriza não rejeição da hipótese estatística de igualdade, entre as estimativas geradas pelos dois modelos.

Esses resultados estão de acordo com os relatos de Matos et al. (1997), que concluíram que os modelos de limiar não foram mais adequados que os modelos lineares, na obtenção de estimativas de parâmetros genéticos, para dados categóricos de características reprodutivas de ovelhas das raças Rambouillet e Finnsheep. Porém, em trabalhos realizados por Ramirez-Valverde et al. (2001), Mwansa et al. (2002) e Marcondes et al. (2005), as estimativas obtidas pelo modelo linear foram de menor magnitude que o modelo de limiar. É importante ressaltar que, no presente trabalho, as características morfológicas possuíam cinco classes ou escores, o que pode ter contribuído para que as estimativas obtidas pelo modelo linear não fossem subestimadas.

Tabela 2. Estatística descritiva das estimativas de herdabilidade para musculosidade, estrutura física e conformação de bovinos da raça Nelore, obtidas a partir de análises bayesianas uni-características, em modelo animal de limiar e linear.

\begin{tabular}{|c|c|c|}
\hline Estatística descritiva & Limiar & Linear \\
\hline & \multicolumn{2}{|c|}{ Musculosidade } \\
\hline Média & 0,14 & 0,12 \\
\hline Moda & 0,14 & 0,11 \\
\hline Mediana & 0,14 & 0,11 \\
\hline \multirow[t]{2}{*}{ Região de credibilidade (95\%) } & 0,09 a 0,20 & 0,06 a 0,18 \\
\hline & \multicolumn{2}{|c|}{ Estrutura física } \\
\hline Média & 0,15 & 0,14 \\
\hline Moda & 0,14 & 0,14 \\
\hline Mediana & 0,15 & 0,14 \\
\hline \multirow[t]{2}{*}{ Região de credibilidade (95\%) } & 0,07 a 0,24 & 0,06 a 0,22 \\
\hline & \multicolumn{2}{|c|}{ Conformação } \\
\hline Média & 0,10 & 0,11 \\
\hline Moda & 0,09 & 0,11 \\
\hline Mediana & 0,10 & 0,11 \\
\hline Região de credibilidade (95\%) & 0,04 a 0,15 & 0,05 a 0,18 \\
\hline
\end{tabular}

Na Tabela 3, são apresentadas as estatísticas descritivas das estimativas de parâmetros genéticos para as características morfológicas musculosidade, estrutura física e conformação, obtidas a partir de análises bayesianas bi-características em modelo animal linearlimiar e linear-linear. Observou-se que, neste caso, a análise incluiu a característica contínua peso aos 210 dias de idade. De modo geral, as estimativas de herdabilidade para as características morfológicas foram semelhantes.

Em relação à característica contínua de peso aos 210 dias de idade, observou-se que a média das estimativas de herdabilidade foi idêntica nas duas análises. No entanto, nas correlações genéticas, observou-se que o modelo linear-limiar proporcionou estimativas de maior magnitude do que o modelo linear. De acordo com Silva et al. (2005), o uso de modelos de limiar pode proporcionar estimativas mais adequadas, pois estes modelos relacionam a resposta observada na escala categórica a uma escala subjacente normal contínua. Resultados de trabalhos de simulação

Tabela 3. Estatística descritiva das estimativas de parâmetros genéticos, para as características categóricas musculosidade (M), estrutura física (E), conformação (C) e característica contínua peso aos 210 dias de idade (P210), de bovinos da raça Nelore, obtidas a partir de análises bayesianas bi-características, em modelo animal linear-limiar e linear-linear ${ }^{(1)}$.

\begin{tabular}{|c|c|c|c|c|}
\hline Análise & Média & Moda & Mediana & $\mathrm{RC}(95 \%)$ \\
\hline & \multicolumn{4}{|c|}{$\begin{array}{l}\text { Modelo linear-limiar } \\
\text { P2 } 10_{1}+\mathrm{M}_{2}\end{array}$} \\
\hline $\mathrm{h}_{1}{ }^{2}$ & 0,19 & 0,19 & 0,19 & 0,14 a 0,23 \\
\hline $\mathrm{h}_{2}{ }^{2}$ & 0,21 & 0,22 & 0,21 & 0,13 a 0,30 \\
\hline \multirow[t]{2}{*}{$r_{g}$} & 0,62 & 0,61 & 0,62 & 0,45 a 0,75 \\
\hline & \multicolumn{4}{|c|}{$\mathrm{P} 210_{1}+\mathrm{E}_{2}$} \\
\hline $\mathrm{h}_{1}{ }^{2}$ & 0,21 & 0,21 & 0,21 & 0,17 a 0,25 \\
\hline $\mathrm{h}_{2}{ }^{2}$ & 0,27 & 0,28 & 0,28 & 0,20 a 0,35 \\
\hline \multirow[t]{2}{*}{$r_{g}$} & 0,94 & 0,99 & 0,96 & 0,80 a 0,99 \\
\hline & \multicolumn{4}{|c|}{$\mathrm{P} 210_{1}+\mathrm{C}_{2}$} \\
\hline $\mathrm{h}_{1}{ }^{2}$ & 0,25 & 0,25 & 0,25 & 0,20 a 0,30 \\
\hline $\mathrm{h}_{2}{ }^{2}$ & 0,29 & 0,31 & 0,31 & 0,07 a 0,40 \\
\hline \multirow[t]{2}{*}{$r_{g}$} & 0,89 & 0,87 & 0,89 & 0,80 a 0,99 \\
\hline & \multicolumn{4}{|c|}{ Modelo linear-linear } \\
\hline $\mathrm{h}_{1}{ }^{2}$ & 0,19 & 0,18 & 0,19 & 0,14 a 0,23 \\
\hline $\mathrm{h}_{2}{ }^{2}$ & 0,20 & 0,19 & 0,19 & 0,13 a 0,27 \\
\hline \multirow[t]{2}{*}{$r_{g}$} & 0,61 & 0,62 & 0,61 & 0,46 a 0,74 \\
\hline & \multicolumn{4}{|c|}{$P 210_{1}+E_{2}$} \\
\hline $\mathrm{h}_{1}{ }^{2}$ & 0,21 & 0,21 & 0,21 & 0,17 a 0,26 \\
\hline $\mathrm{h}_{2}{ }^{2}$ & 0,29 & 0,28 & 0,29 & 0,20 a 0,37 \\
\hline \multirow[t]{2}{*}{$r_{g}$} & 0,86 & 0,90 & 0,87 & 0,76 a 0,95 \\
\hline & \multicolumn{4}{|c|}{$\mathrm{P} 210_{1}+\mathrm{C}_{2}$} \\
\hline $\mathrm{h}_{1}^{2}$ & 0,25 & 0,25 & 0,25 & 0,20 a 0,29 \\
\hline $\mathrm{h}_{2}{ }^{2}$ & 0,33 & 0,33 & 0,33 & 0,25 a 0,42 \\
\hline$r_{g}$ & 0,85 & 0,85 & 0,85 & 0,78 a 0,92 \\
\hline
\end{tabular}

${ }^{(1)} \mathrm{RC}$ : região de credibilidade a $95 \% ; \mathrm{h}^{2}$ : herdabilidade; $\mathrm{r}_{\mathrm{g}}$ : correlação genética; os valores subscritos 1 e 2 indicam, respectivamente, características 1 e 2 da análise bi-característica. 
(Meijering \& Gianola, 1985; Hoeschele \& Gianola, 1988) mostraram vantagens dos modelos de limiar, em relação aos modelos lineares. No entanto, as análises com dados de campo não permitiram afirmar que os métodos de limiar são mais adequados que os métodos lineares, a não ser, teoricamente. Porém, Varona et al. (1999) utilizaram dados de grandes rebanhos em modelo animal e observaram melhores resultados do modelo de limiar sobre o linear, em análises bi-características, o que não ocorreu para análises uni-características.

Observou-se que as estimativas de parâmetros genéticos, para as características categóricas de musculosidade, estrutura física e conformação, obtidas a partir de análises bayesianas bi-características em modelo animal limiar-limiar e modelo animal linear-linear, foram semelhantes para ambos os modelos (Tabela 4). Em relação à região de credibilidade, observou-se que as amplitudes foram muito próximas.

A vantagem do modelo linear foi a maior velocidade de processamento das análises. De acordo com Marcondes et al. (2005), as análises neste modelo podem ser preferidas, quando houver um grande arquivo de

Tabela 4. Estatística descritiva das estimativas de parâmetros genéticos, para musculosidade (M), estrutura física (E) e conformação $(\mathrm{C})$ de bovinos da raça Nelore, obtidas a partir de análises bayesianas bi-características, em modelo animal de limiar-limiar e linear-linear ${ }^{(1)}$.

\begin{tabular}{|c|c|c|c|c|}
\hline Análise & Média & Moda & Mediana & $\mathrm{RC}(95 \%)$ \\
\hline & \multicolumn{4}{|c|}{ Modelo de limiar-limiar } \\
\hline & \multicolumn{4}{|c|}{$\mathrm{M}_{1}+\mathrm{E}_{2}$} \\
\hline $\mathrm{h}_{1}^{2}$ & 0,15 & 0,15 & 0,15 & 0,08 a 0,22 \\
\hline $\mathrm{h}_{2}^{2}$ & 0,18 & 0,16 & 0,17 & 0,09 a 0,28 \\
\hline \multirow[t]{2}{*}{$r_{g}$} & 0,18 & 0,23 & 0,20 & $-0,23$ a 0,50 \\
\hline & \multicolumn{4}{|c|}{$\mathrm{M}_{1}+\mathrm{C}_{2}$} \\
\hline $\mathrm{h}_{1}^{2}$ & 0,17 & 0,18 & 0,17 & 0,11 a 0,26 \\
\hline $\mathrm{h}_{2}{ }^{2}$ & 0,18 & 0,18 & 0,18 & 0,09 a 0,27 \\
\hline \multirow[t]{2}{*}{$r_{g}$} & 0,75 & 0,74 & 0,76 & 0,57 a 0,88 \\
\hline & \multicolumn{4}{|c|}{$\mathrm{E}_{1}+\mathrm{C}_{2}$} \\
\hline $\mathrm{h}_{1}^{2}$ & 0,18 & 0,15 & 0,17 & 0,10 a 0,28 \\
\hline $\mathrm{h}_{2}^{2}$ & 0,14 & 0,15 & 0,15 & 0,05 a 0,22 \\
\hline \multirow[t]{3}{*}{$r_{g}$} & 0,56 & 0,53 & 0,57 & 0,26 a 0,86 \\
\hline & \multicolumn{4}{|c|}{ Modelo linear-linear } \\
\hline & \multicolumn{4}{|c|}{$\mathrm{M}_{1}+\mathrm{E}_{2}$} \\
\hline $\mathrm{h}_{1}^{2}$ & 0,13 & 0,13 & 0,13 & 0,07 a 0,19 \\
\hline $\mathrm{h}_{2}^{2}$ & 0,15 & 0,12 & 0,15 & 0,09 a 0,25 \\
\hline$r_{g}$ & 0,19 & 0,23 & 0,21 & $-0,21$ a 0,50 \\
\hline & \multicolumn{4}{|c|}{$\mathrm{M}_{1}+\mathrm{C}_{2}$} \\
\hline $\mathrm{h}_{1}^{2}$ & 0,15 & 0,16 & 0,15 & 0,09 a 0,22 \\
\hline $\mathrm{h}_{2}^{2}$ & 0,16 & 0,16 & 0,16 & 0,09 a 0,24 \\
\hline \multirow[t]{2}{*}{$r_{g}$} & 0,76 & 0,81 & 0,77 & 0,58 a 0,89 \\
\hline & \multicolumn{4}{|c|}{$\mathrm{E}_{1}+\mathrm{C}_{2}$} \\
\hline $\mathrm{h}_{1}{ }^{2}$ & 0,13 & 0,14 & 0,13 & 0,07 a 0,20 \\
\hline $\mathrm{h}_{2}{ }^{2}$ & 0,15 & 0,12 & 0,14 & 0,09 a 0,24 \\
\hline$r_{g}$ & 0,59 & 0,60 & 0,60 & 0,24 a 0,85 \\
\hline
\end{tabular}

${ }^{(1)} \mathrm{RC}$ : região de credibilidade a $95 \% ; \mathrm{h}^{2}$ : herdabilidade; $\mathrm{r}_{\mathrm{g}}$ : correlação genética; os valores subscritos 1 e 2 indicam, respectivamente características 1 e 2 da análise bi-característica. dados e muitos registros de produção. Neste trabalho, o uso de modelos de limiar apresentou maior demanda computacional como o maior tempo gasto na obtenção das estimativas de parâmetros genéticos, e este fato foi proporcionado, provavelmente, pela maior demanda de ciclos da cadeia de Gibbs, para se alcançar a convergência.

As correlações de posto ou correlações de Spearman, para as estimativas de DEPs para musculosidade, estrutura física e conformação, considerando-se as análises uni-características em modelo animal linear e de limiar, foram acima de 99\%, o que indica que, praticamente, não houve mudança na classificação dos animais, conforme o modelo adotado. $\mathrm{O}$ mesmo ocorreu nas análises bi-características (dados contínuos e categóricos), em modelo animal linear-limiar e linearlinear e nas análises bi-características (dados categóricos) em modelo animal linear-linear e limiarlimiar, em que as correlações de posto também foram maiores que $99 \%$. Assim, verificou-se que o uso dos diferentes modelos de avaliação genética, propostos neste trabalho, não modificou a classificação dos animais.

Estes resultados corroboram os de Marcondes et al. (2005), que concluíram que não houve diferença na classificação dos animais, quando os valores genéticos foram preditos pelos modelos bayesianos de limiar ou linear. Devido ao menor tempo computacional, os autores recomendaram o uso dos modelos lineares para dados categóricos. Jamrozik et al. (1991) estudaram cinco características de conformação, em bovinos da raça Holandesa, e observaram que a classificação dos touros foi a mesma para ambos os modelos.

\section{Conclusão}

1. Não há diferença entre o modelo linear e o de limiar, na obtenção de estimativas de herdabilidades e correlações genéticas, de características categóricas morfológicas multinomiais.

2. Na avaliação genética, o uso do modelo de limiar ou linear, não influencia a classificação dos animais, quanto aos valores genéticos preditos, o que favorece a utilização de ambos os modelos em programas de melhoramento genético.

\section{Agradecimentos}

Ao Conselho Nacional de Desenvolvimento Científico e Tecnológico, pelo apoio financeiro; à Embrapa Arroz e Feijão, ao Departamento de Genética e Melhoramento 
da Universidade Estadual Paulista e à Escola de Veterinária da Universidade Federal de Goiás, pelo apoio técnico-científico; ao Dr. José Benedito de Freitas Trovo, pela revisão deste trabalho.

\section{Referências}

BENNETT, G.L.; GREGORY, K.E. Genetic (co)variances for calving difficulty score in composite and parental populations of beef cattle: I. Calving difficulty score, birth weight, weaning weight, and postweaning gain. Journal of Animal Science, v.79, p.45-51, 2001. CARDOSO, F.F.; CARDELLINO, R.A.; CAMPOS, L.T. Fatores ambientais sobre escores de avaliação visual à desmama em bezerros Angus criados no Rio Grande do Sul. Revista Brasileira de Zootecnia, v.30, p.318-325, 2001.

CARNIER, P.; ALBERA, A.; DAL ZOTTO, R.; GROEN, A.F.; BONA, M.; BITTANTE, G. Genetic parameters for direct and maternal calving ability over parities in Piedmontese cattle. Journal of Animal Science, v.78, p.2532-2539, 2000.

DAL FARRA, R.A.; ROSO, V.M.; SCHENKEL, F.S. Efeitos de ambiente e de heterose sobre o ganho de peso do nascimento ao desmame e sobre os escores visuais ao desmame de bovinos de corte. Revista Brasileira de Zootecnia, v.31, p.1350-1361, 2002. (Suplemento).

GIANOLA, D.; FOULLEY, J.L. Sire evaluation for ordered categorical data with a threshold model. Genetics Selection Evolution, v.15, p.201-224, 1983.

GIANOLA, D.; SORENSEN, D. Likelihood, Bayesian, and MCMC methods in quantitative genetics. New York: SpringerVerlag, 2002.740p.

HOESCHELE, I.; GIANOLA, D. Bayesian versus maximum quasilikelihood methods for sire evaluation with categorical data. Journal of Dairy Science, v.72, p.1569-1577, 1988.

JAMROZIK, J.; SCHAEFFER, L.R.; BURNSIDE, E.B.; SULLIVAN, B.P. Threshold models applied to Holstein conformation traits. Journal of Dairy Science, v.74, p.3196-3201, 1991.

KIPPERT, C.J.; RORATO, P.R.N.; CAMPOS, L.T.; BOLIGON, A.A.; WEBER, T.; GHELLER, D.G.; LOPES, J.S. Efeito de fatores ambientais sobre escores de avaliação visual à desmama e estimativa de parâmetros genéticos, para bezerros da raça Charolês. Ciência Rural, v.36, p.579-585, 2006.

KOURY FILHO, W. Escores visuais e suas relações com características de crescimento em bovinos de corte. 2005. 80p. Tese (Doutorado) - Universidade Estadual Paulista, Jaboticabal.

LÔBO, R.B. Programa de melhoramento genético da raça Nelore. Ribeirão Preto: Finep, 1996. 100p.

LUO, M.F.; BOETTCHER, P.J.; SCHAEFFER, L.R.; DECKKERS, J.C.M. Bayesian inference for categorical traits with an application to variance component estimation. Journal of Dairy Science, v.84, p.694-704, 2001.

LUO, M.F.; BOETTCHER, P.J.; SCHAEFFER, L.R.; DEKKERS, J.C.M. Estimation of genetic parameters of calving ease in first and second parities of Canadian Holsteins using Bayesian methods. Livestock Production Science, v.74, p.175-184, 2002.

MARCONDES, C.R.; PANETO, J.C.C.; SILVA, J.A.V.; OLIVEIRA, H.N.; LÔBO, R.B. Comparação entre análises para permanência no rebanho de vacas Nelore utilizando modelo linear e modelo de limiar. Arquivo Brasileiro de Medicina Veterinária e Zootecnia, v.57, p.234-240, 2005.

MATOS, C.A.P.; THOMAS, D.L.; GIANOLA, D.; TEMPELMAN, R.J.; YOUNG, L.D. Genetic analysis of discrete reproductive traits in sheep using linear and nonlinear models: I. Estimation of genetic parameters. Journal of Animal Science, v.75, p.76-87, 1997.

MEIJERING, A.; GIANOLA, D. Linear versus nonlinear methods of sire evaluation for categorical traits: a simulation study. Genetics Selection Evolution, v.17, p.115-131, 1985.

MORENO, C.; SORENSEN, D.; GARCÍA-CORTÉS, L.A.; VARONA, L.; ALTARRIBA, J. On biased inferences about variance components in the binary threshold model. Genetics Selection Evolution, v.29, p.145-160, 1997.

MWANSA, P.B.; CREWS, D.H.J.; WILTON, J.W.; KEMP, R.A. Multiple trait selection for maternal productivity in beef cattle. Journal of Animal Breeding Genetics, v.119, p.391-399, 2002.

PHOCAS, F.; LALOË, D. Evaluation models and genetic parameters for calving difficulty in beef cattle. Journal of Animal Science, v.81, p.933-938, 2003.

RAMIREZ-VALVERDE, R.; MISZTAL, I.; BERTRAND, J.K. Comparison of threshold vs linear and animal vs sire models for predicting direct and maternal genetic effects on calving difficulty in beef cattle. Journal of Animal Science, v.79, p.333-338, 2001.

SAS INSTITUTE. SAS OnlineDoc 9.1.3. Cary: SAS Institute, 2004.

SILVA, J.A.V.; DIAS, L.T.; ALBUQUERQUE, L.G. Estudo genético da precocidade sexual de novilhas em um rebanho Nelore. Revista Brasileira de Zootecnia, v.34, p.1568-1572, 2005.

SORENSEN, D.A.; ANDERSEN, S.; GIANOLA, D.; KORSGAARD, I. Bayesian inference in threshold models using Gibbs sampling. Genetics Selection Evolution, v.27, p.229-249, 1995.

VAN KAAM, J.B.C.H.M. Gibanal 2.9: analyzing program for Markov chain Monte Carlo sequences. Wageningen: Wageningen Agricultural University, 1998.

VAN TASSELL, C.P.; VAN VLECK, L.D.; GREGORY, K.E. Bayesian analysis of twinning and ovulation rates using a multipletrait threshold model and Gibbs sampling. Journal of Animal Science, v.76, p.2048-2061, 1998.

VARONA, L.; MISZTAL, I.; BERTRAND, J.K. Threshold-linear versus linear-linear analysis of birth weight and calving ease using an animal model: I. Variance component estimation. Journal of Animal Science, v.77, p.1994-2002, 1999.

WEIGEL, K.A.; REKAYA, R. Genetic parameters for reproductive traits of Holstein cattle in California and Minnesota. Journal of Dairy Science, v.83, p.1072-1080, 2000.

$\overline{\text { Recebido em } 20 \text { de janeiro de } 2008 \text { e aprovado em } 30 \text { de junho de } 2008}$ 